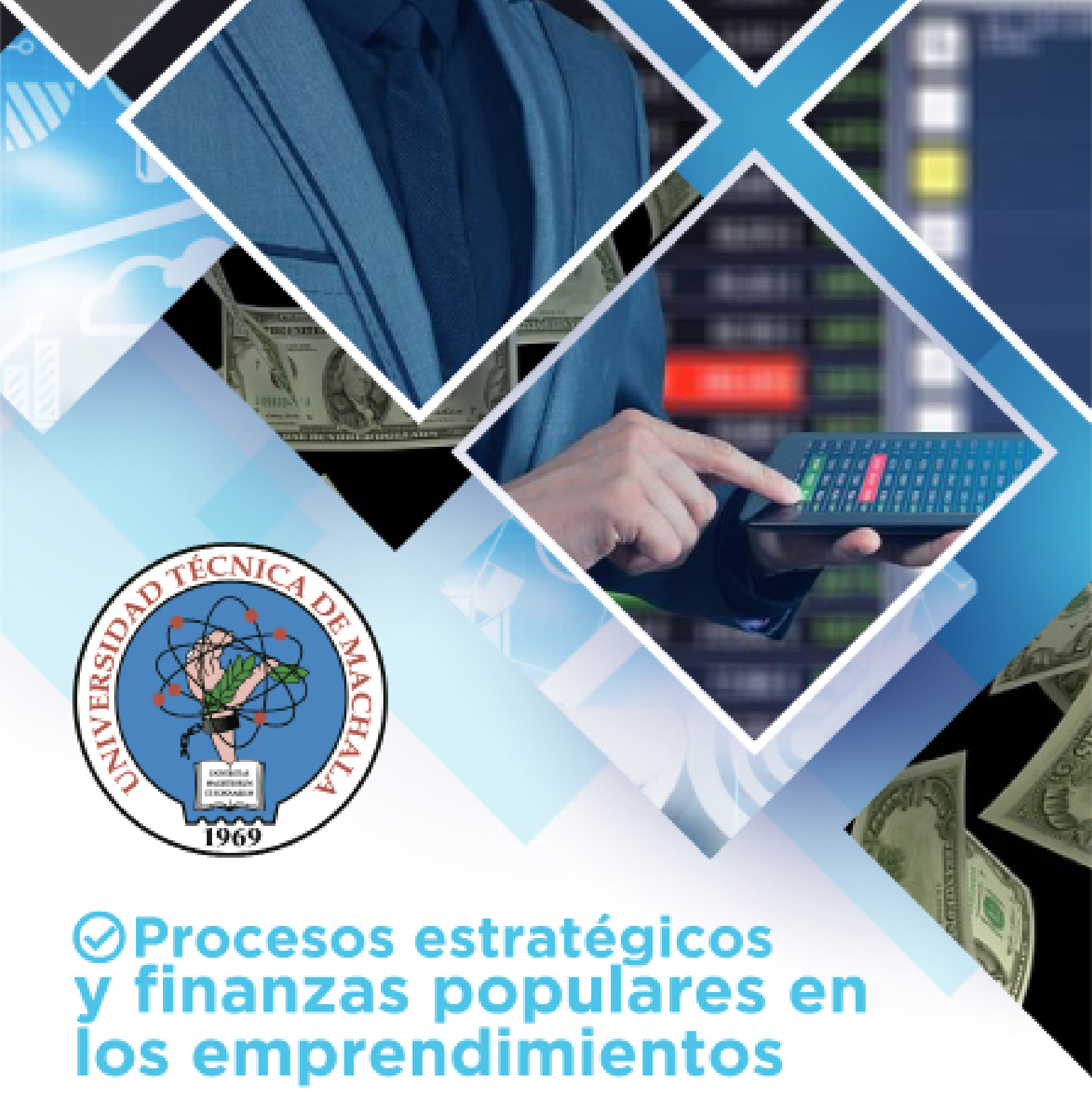

Lady León-Serrano Compiladora

colocción: Semana de la ciencia $=y_{-1} \mathrm{~d}_{\mathrm{Tral}}$ 


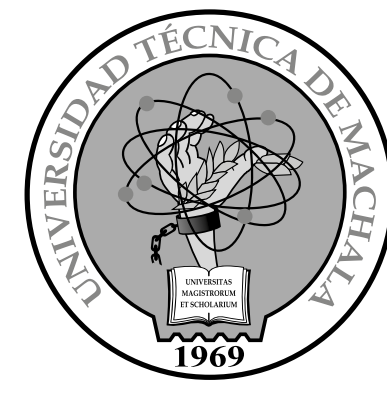

(c) (i) ()

Ediciones UTMACH 86 pág: $21 \times 25,5 \mathrm{~cm}$

Colección Semana de la Ciencia

Título: Procesos estratégicos y finanzas populares en los emprendimientos - Lady León Serrano (Compiladora)

Primera edición - junio 2021

ISBN: 978-9942-24-144-3

CDD 336

1. Economía,

2. Empresa y gestión

--Temas relacionados

Publicación PDF 


\section{Procesos estratégicos y finanzas populares en los emprendimientos}


Lady León-Serrano

COMPILADORA 
César Quezada Abad - Rector

Amarilis Borja Herrera - Vicerrector Académico

Jhonny Pérez Rodríguez - Vicerrector Administrativo

Luis Brito Gaona

Director de Investigación

(c) Ediciones UTMACH

Colección Semana de la Ciencia

Título original:

Procesos estratégicos y finanzas populares en los emprendimientos

ISBN: 978-9942-24-144-3

DOI: http://doi.org/10.48190/9789942241443

Libro con revisión de pares ciegos especializados

(c) Lady León-Serrano

(Compiladora)

(c) Autores de capítulos

Karina Lozano Zambrano Jefe editor / Diseño y edición editorial Edison Mera León - Diseño de portada

Fernanda Tusa Jumbo - Corrector de estilos Jorge Maza-Cordova - Asesor tecnológico Karla Ibañez y Cyndi Aguilar - Equipo de difusión

Primera edición Junio 2021 Machala-Ecuador

Universidad Técnica de Machala - UTMACH Correo: editorial@utmachala.edu.ec Licencia Creative Commons Atribución-NoComercial-CompartirIgual 4.0 Internacional (CC BY-NC-SA 4.0). 


\section{Contenido}

Gestión por procesos como herramienta estratégica en las pequeñas y medianas empresas

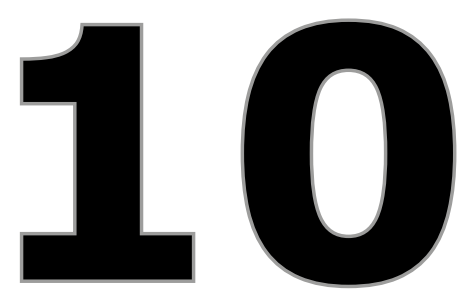

Finanzas populares y solidarias como alternativas para el desarrollo en los emprendimientos de eocnomía popular y solidaria en la ciudad de Machala, 2019

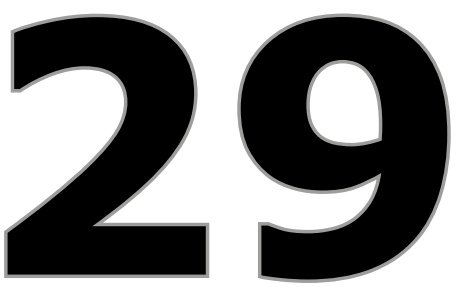

Los emprendedores y el poder de adaptación en los tiempos de pandemia
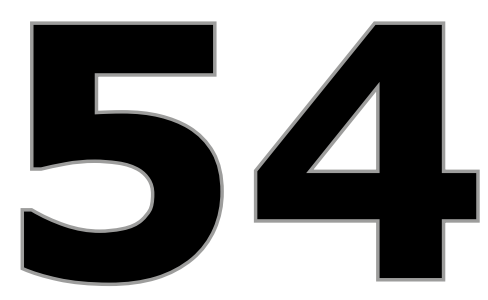

Pág. 


\section{Introducción}

El desarrollo de los emprendimientos es considerado un factor estratégico para el avance económico, comercial y solidario de los países, el aporte de nuevos negocios con productividad ha generado empleo y valor agregado a las economías, sin embargo, los diversos problemas de competitividad que enfrentan afecta la capacidad de sobrevivencia, sin duda el progreso de los mercados conformados en gran parte por las pequeñas y medianas empresas implica adaptación de instrumentos globales que permitan perfeccionar las habilidades gerenciales y organizacionales desde los procesos vinculantes con buenas prácticas y mejora continua.

En este sentido, la organización y permanencia del núcleo emprendedor permite hacer frente ante los factores externos, creando alternativas con elementos estratégicos, enfoques sistemáticos confiables y característicos en la toma de decisiones, en base a la intensionalidad de crear incentivos favorables propios de los actores sociales, aprovechando las fortalezas del entorno con programas de apoyo financiero, no financiero, acceso a nuevas fuentes de aprovisionamiento y condiciones socioeconómicas.

Consecuentemente, la transformación de los emprendimientos desde una masificación internacional y nacional ha requerido del impulso de políticas públicas con tratamientos de gestión y control interno, más allá de un margen estratégico de comercialización, se asocia con las finanzas populares y solidarias en la plena capacidad de planificar y regular el interés general, asumiendo un rol protagónico de intermediación y prestación de servicios financieros entre sí. Así, el fomento de la producción tiene un sentido cooperativo, con igualdad de derechos y participación. 
El impacto de la dinámica del conocimiento e innovación de los productos y servicios demanda cada vez la satisfacción de necesidades locales. El comportamiento del comercio global, competidores y clientes, constantemente presentan variaciones en los planes estratégicos con un pensamiento de comercio justo, equitativo y sostenibilidad en el tiempo.

Los factores externos llevan a profundizar la resiliencia de las perqueñas y medianas empresas, aquellos emprendedores trastocados por la pandemia generada por el Covid-19 junto a los momentos de incertidumbre han puesto en marcha nuevas habilidades en el uso de recursos tecnológicos y empoderamiento social, marcando la crisis como una oportunidad.

El aporte intelectual de la presente obra, parte de la Colección Semana de la Ciencia de la Editorial Utmach, contribuye en la difusión de conocimientos sobre los procesos estratégicos y finanzas populares, y la claridad de emprender en tiempos de pandemia desde la innovación tecnológica sustentado en las bases teóricas y la cuantificación de datos para la ejemplaridad reflexiva del lector en el alcance de la visualización y posicionamiento internacional. 



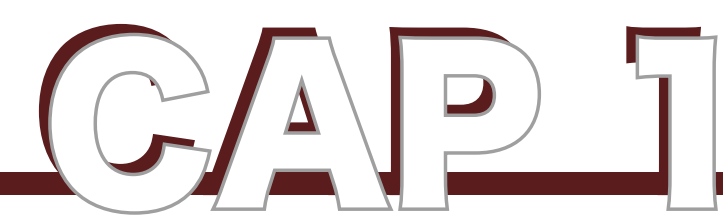

Gestión por procesos como herramienta estratégica en las pequeñas y medianas empresas

Vargas Jiménez Mónica del Carmen

Farez Arias Miriam Rocio

conzález Illescas Mayiya Lisbeth

Melean Romero Rosana 


\section{AUTORES}

\section{Vargas Jiménez Mónica del Carmen}

Investigadora Externa-Universidad Técnica de Machala / Magister en Auditoría Integral en la Universidad Técnica Particular de Loja, UTPL. Doctoranda en Ciencias Sociales Mención Gerencia, en la Universidad del Zulia-Venezuela. Miembro del Grupo de investigación COMINDEP Nro. 025 - Dominio Economía Empresa y Productividad adscrito al Centro de Investigación - UTMACH. movaji@hotmail.com

\section{Farez Arias Miriam Rocio}

Profesora titular de la Universidad Técnica de Machala. Magister en Ingeniería de Software de la Universidad de las Fuerzas Armadas, ESPE.

Miembro del Grupo de investigación COMINDEP Nro. 025 - Dominio Economía Empresa y Productividad adscrito al Centro de Investigación - UTMACH.

mrfarez@utmachala.edu.ec

\section{González Illescas Mayiya Lisbeth}

Economista, Universidad de Cuenca; Magister en Comercio Internacional, Universidad de Alicante, España; Doctora en Empresa, Economía y Sociedad, Universidad de Alicante, España; coordinadora del grupo de investigación Comercio Internacional y Desarrollo Productivo, COMINDEP, UTMACH; directora del proyecto "Análisis de las estrategias para la competitividad empresarial en los mercados dinámicos internacionales", Res. 359-2020.

mlgonzalez@utmachala.edu.ec

\section{Melean Romero Rosana}

Posdoctora por la Universidad Nacional Toribio Rodríguez de Mendoza (UNTRM). Doctora en Ciencias Sociales, mención Gerencia. Magíster en Gerencia de empresas, Mención Gerencia Industrial. Licenciada en Administración. Profesora Titular e Investigadora de la Universidad del Zulia, Maracaibo, Venezuela. Directora de la Revista Venezolana de Gerencia (Revista Scopus Q3). Scopus Author ID: 22954427400; ORCID: https://orcid.org/0000-0001-8779-738X rosanamelean@gmail.com 


\section{Gestión por procesos como herramienta estratégica en las pequeñas y medianas empresas ${ }^{1}$}

\section{Resumen}

El objetivo de la investigación fue identificar desde un enfoque teórico la gestión por procesos como herramienta estratégica para la satisfacción del cliente (interno/ externo) de pequeñas y medianas empresas. La investigación es documental-descriptiva, de orden cualitativa, parte de una revisión teórica documental, apoyada en búsquedas especializadas en bases de datos reconocidas internacionalmente, de preferencia: Scopus, Redalyc, Dialnet y Scielo, otras de acceso abierto, y demás fuentes secundarias de corriente regional. Los materiales recopilados fueron organizados y sistematizados para extraer de ellos información requerida para soportar este trabajo. Los resultados del recorrido teórico sobre la gestión por procesos, aproximan a su importancia en un entorno dinámico que fracciona pensamientos tradicionales asociados a dimensiones tecnológicas y de productividad; la gestión por procesos orienta su accionar a la implementación de buenas prácticas, la mejora continua y la satisfacción del cliente con criterios de eficiencia y agregación de valor. En el caso de las pequeñas y medianas empresas, se ha propuesto mejorar sus procesos desde una visión integradora de su filosofía de gestión; lo que implica, el despliegue de innovaciones organizativas a nivel interno, mostrando estructuras funcionales con capacidad gerencial y eficiencia en las operaciones. Como conclusión, se establece que la gestión por procesos como herramienta estratégica gana importancia en las pequeñas y medianas empresas por la organización lógica de sus recursos y la

\footnotetext{
${ }^{1 *}$ Esta ponencia recoge resultados avances teóricos sobre la Gestión por procesos, que contribuye al Grupo de Investigación COMINDEP-UTMACH y, al proyecto de Tesis inserta en la línea Finanzas del Programa doctoral Ciencias Sociales mención Gerencia de Empresas de la División de Estudios para Graduados de la Facultad de Ciencias Económicas y Sociales de la Universidad del Zulia.
}

DOI: http://doi.org/10.48190/9789942241443.1

IR AL INDICE

12 
adaptabilidad ante estructuras tradicionales. Se plantea una gestión interfuncional generadora de valor y la disminución de actividades que no aporten a la eficiencia interna y a la satisfacción del cliente.

Palabras clave: gestión por procesos, proceso estratégico, proceso operativo, proceso de apoyo.

\section{Introducción}

Antesala del siglo XXI, las empresas tienen el reto de adaptarse a escenarios dinámicos y complejos, donde es necesario el equilibrio de los factores de oferta y demanda del mercado, esto significa el implementar herramientas que se orienten hacia el cliente interno como externo, en busca de la satisfacción de sus necesidades, pues sus alternativas de supervivencia estan limitadas por su estructura, distribución de tareas, personas, clima laboral, tecnología, competitividad e innovación. Para mantener dicho dinamismo Gonzalez, et al., (2019) proponen un enfoque a los resultados institucionales, considerando la gestión basada en procesos, herramienta que surge de la evolución del pensamiento administrativo, que desde lo vertical trata de comprender el entorno globalizador, pues no trata de eliminar los esquemas tradicionales, sino mejorar o cambiar estrategias de funcionalidad, tomando como centro los procesos, buscando desplegar desde ellos criterios de eficiencia, eficacia y calidad (García et al., 2019; Hernández et al., 2018), que deben derivar de la implementación de técnicas, herramientas y ontologías de gestión (Morales, 2014), como buenas prácticas para mejorar la gestión organizacional (Tatić et al., 2018).

Desde algunos aportes como el de Gonzalez et al., (2019), el enfoque por procesos asume relevancia en el sector empresarial, al poner énfasis en la gestión de las interrelaciones $(\mathrm{s} / \mathrm{n}$ ) de actividades, lo que ha significado conocer vínculaciones en los procesos de planificación, coordinación, ejecución, control y la mejora contínua. En efecto, las prácticas de gestión ejercidas por los agentes internos, demuestran su capacidad para generar nuevas ideas e incorporar valor a los elementos de entrada y salida, los mismo que alimentan a los procesos y otorgan satisfacción a un cliente. Ahora bien, en la postmodernidad, este nuevo enfoque administrativo basado en los procesos se caracteriza por su visión integral en los niveles estratégicos, operativos

Gestión por procesos como herramienta estratégica en las pequeñas y medianas empresas

INICIO DEL CAPÍTULO 
y de apoyo, así involucra un conjunto de recursos que interrelaciona tareas, procedimientos en cada actividad de la empresa (Estupiñan \& Batista, 2018).

Medina \& Nogueira (2018) justifican que son limitadas las empresas diseñadas y gestionadas por proceso, a causa de su rígida cultura empresarial que obstaculiza la alineación de los procesos con la estrategia de satisfacción al cliente, creación de valor y capacidad de respuesta. Con una mirada, hacia las pequeñas y medianas empresas, el reto es mayor, debido a que se caracterizan por su estructura familiar rígida, por ende, limita su competitividad en el mercado nacional e internacional, naturalmente, requieren replantear estrategias de gestión con fines de alcanzar la sostenibidad de sus actividades económicas (Mila et al., 2019), por supuesto, depende de la efectividad de sus procesos empresariales para que estén alineados con la estrategia, misión y objetivos institucionales (Medina et al., 2009).

Así tambien, Avolio et al., (2011) y Beltran (2006) establece la existencia de diversos modelos para el desarrollo de las micro y pequeñas empresas (MyPEs), logrando identificar cinco factores de influencia como: administrativos, operativos, estratégicos, externos y personales, los cuales limitan la consolidación y el crecimiento de los empresarios de las MyPEs. Es entonces relevante estudiar los aportes sobre la gestión por procesos como herramienta estratégica que con una mirada holística que podría mitigar riesgos de sostenibilidad empresarial.

En ese sentido, con el aparecimiento de la gestión por procesos, no significó el abandono de las teorías tradicionales, sino su actualización, pues se trataba de una nueva estrategia administrativa para gestionar recursos, consolidando, el nuevo rol de la gerencia para comprender, y dirigir los cambios de los procesos de una empresa (Harrington, 1993); una nueva ruta para la optimización de los recursos de entrada y salida. La gestión por procesos, se ha constituido un reto para las empresas, que trae consigo el ser eficiente, eficaz y productiva conforme la estrategia, misión y objetivo organizacional (Cabrera et al., 2015). La visión holística de la gestión por procesos toma de parámetro, al proceso y con ello, lo que llamó Porter (1987) el encadenamiento de valor ${ }^{2}$.

2*Porter (1987 ed. rev.2006) La cadena de valor está integrada a un flujo más grande de actividades al que doy el nombre de sistema de valores...Los proveedores cuentan con cadenas de valores (valor ascendente) que crea y entrega los insumos utilizados en ellas. No sólo suministran un producto, sino que también pueden influir en el desempeño que la compañía tiene en muchas otras áreas (p.33). 
La perspectiva administrativa, en un inicio corresponde a un enfoque funcional que desde lo clásico y neoclásico se orientaba a la departamentalización, donde el "proceso", como parte del sistema empresarial existe con su concepto de transformación e incorporación de valor (Medina et al., 2019) que reconoce las formas de gestión de la organización, con la vinculación de un elemento importante como la estructura organizacional.

A fin de dar cumplimiento al objetivo de investigación caracterizar la gestion por procesos como herramienta estratégica para la satisfacción de cada cliente (interno/externo) de las pequeñas y medianas empresas, se efectuó un estudio documental - descriptivo que parte de una revisión bibliográfica con la finalidad de establecer los avances teóricos sobre la gestión por procesos, y a la vez identificar aspectos que denoten su importancia como herramienta estratégica en el contexto de la pequeñas y medianas empresas.

\section{Materiales y métodos}

La investigación es documental-descriptiva, de orden cualitativa, parte de una revisión teórica documental. La búsqueda realizada, se apoya en bases de datos reconocidas internacionalmente, de preferencia: Scopus, Redalyc, Dialnet y SciELO, otras de acceso abierto, y demás fuentes secundarias de corriente regional. Se rastrearon cerca de treinticuatro documentos que permitieron describir y comprender aspectos centrales de la gestión por procesos. Se asumieron criterios de inclusión y selección de documentos: idioma (español), artículos relacionados con la temática planteada: gestion por procesos bajo criterios de herramienta estratégica, rastreando también documentos que aborden su aceptación en las pequeñas y medianas empresas.

Se desarrolló una lectura comprensiva que consolida ideas principales, que formaron parte de la narración. Con aplicación de este método, se obtuvo como resultado los avances teóricos más relevantes sobre la gestión por procesos y su importancia en las pequeñas empresas, conforme se presenta en los siguientes párrafos. 


\section{Gestión por procesos}

Para comprender ¿Qué es la gestión por procesos? se debe partir del concepto de "proceso". Para ello, Harrington (1991) la define como aquella actividad o conjunto de actividades que toma una entrada, para agregarle valor y facilita una salida para un cliente interno o externo. El proceso requiere ser constituido desde lo secuencial para poder interrelacionarlo en sus procesos de entrada y salida que debe contener valor. Para Blázquez \& Amato (2016) el aporte de mayor significancia de los procesos corresponde la incorporación de una mirada de trazabilidad en la organización a través de los procesos organizacionales en función de la cadena de valor (p.90) a esto refiere enfatizar a los procesos en las decisiones estratégicas y competitivas de la gestión empresarial.

Los procesos han requerido modificaciones, pero en un inicio estuvieron direccionados hacia la mejora de actividades y los procesos, inobservando la exigencia de alineacion hacia la estrategia, en ese sentido la función del proceso es producir respuestas añadiendo valor a las entradas; en consecuencia, para Cabrera et al., (2015), la adaptación de la gestión por procesos en el contexto empresarial corresponde al medio de aprovechamiento de los recursos y capacidades para la sostenibilidad en el mercado, más no, como aquel logro alcanzado por una organización al ser reconocida por su competitividad empresarial.

A lo expuesto en párrafos anteriores, se construyó un corpus textual (Ver Tabla 1) sobre la variable en estudio "gestión por procesos", los mismos que exponen proposiciones en beneficio del sector empresarial, se puede incluir que "la gestión por procesos, es uno de los conceptos modernos de direccionamiento, que ha resultado como apoyo a la necesidad que tienen las organizaciones de encaminar sus esfuerzos para la construcción de una nueva estructura administrativa (Hernández et al., 2016; Mallar, 2010; Ochoa et al., 2012; Maldonado, 2013); dicha estructura debe tener una orientación horizontal, que busca la alineación de los procesos con la estrategia, misión y objetivos, en su interrelación con direccionamieto hacia el cliente, el aporte de valor, como tambien la habilidad de respuesnta (Medina \& Nogueira, 2019). 
Tabla 1. Aportes sobre la gestión por procesos

\begin{tabular}{|c|c|}
\hline Fuente & Planteamiento \\
\hline $\begin{array}{l}\text { Armistead, C., \& Machin, S. } \\
(1998)\end{array}$ & $\begin{array}{l}\text { Facilita a una organización posicionar la productividad } \\
\text { del servicio frente a la calidad y comprender mejor los } \\
\text { complejos vínculos entre la satisfacción del cliente, a } \\
\text { través del desempeño general de los procesos y la produc- } \\
\text { tividad }\end{array}$ \\
\hline $\begin{array}{l}\text { Medina León, A., Nogueira Rive- } \\
\text { ra, D., \& Hernández Nariño, A. } \\
(2009)\end{array}$ & $\begin{array}{l}\text { Gestiona toda la organización basándose en los procesos. } \\
\text { Percibe la organización como un sistema interrelacionado. }\end{array}$ \\
\hline Mallar, Miguel Ángel. (2010) & $\begin{array}{l}\text { Nuevo concepto de estructura organizativa que considera } \\
\text { que toda organización se puede concebir como una red de } \\
\text { procesos interrelacionados o interconectados. }\end{array}$ \\
\hline Bravo, J. (2011) & $\begin{array}{l}\text { Es una disciplina de gestión que ayuda a la dirección de } \\
\text { la empresa a identificar, representar, diseñar, formalizar, } \\
\text { controlar, mejorar y hacer más productivos los procesos } \\
\text { de la organización para lograr la confianza del cliente. }\end{array}$ \\
\hline $\begin{array}{l}\text { Ochoa Baque, A., Herrera Leon, J., } \\
\text { \& Mosqera Aguirre, J. (2012). }\end{array}$ & $\begin{array}{l}\text { Es la representación de gestionar toda la organización } \\
\text { fundándose en los Procesos, estos se congregan en la vigi- } \\
\text { lancia del resultado de estos y no en las tareas o acciones }\end{array}$ \\
\hline Maldonado Mina, D. O. (2013) & $\begin{array}{l}\text { Es la sistematización de la gestión en base a un conjunto } \\
\text { de procesos interrelacionados, donde cada uno de ellos } \\
\text { cumple una función específica de generar valor a los insu- } \\
\text { mos, con la finalidad de obtener un producto y/o servicio } \\
\text { de calidad que satisfaga las necesidades de los clientes. }\end{array}$ \\
\hline $\begin{array}{l}\text { Ruiz-Fuentes, Daysi, \& Alma- } \\
\text { guer-Torres, Rosa Mercedes, \& } \\
\text { Torres-Torres, Isabel Cristina, \& } \\
\text { Hernández-Peña, Alejandro Miguel } \\
(2014) .\end{array}$ & $\begin{array}{l}\text { Es una forma de conducir o administrar una organiza- } \\
\text { ción, concentrándose en el valor agregado para el cliente } \\
\text { y las partes interesadas. }\end{array}$ \\
\hline $\begin{array}{l}\text { Sánchez Ruiz, L., Blanco Rojo, B., } \\
\text { Pérez Labajos, C. Á., \& Serrano } \\
\text { Bedia, A. M. (2014). }\end{array}$ & $\begin{array}{l}\text { Nueva forma de organización y administración empre- } \\
\text { sarial destinada a todo tipo de empresas que persigue, } \\
\text { simultáneamente, el aumento de la eficiencia empresarial } \\
\text { y el incremento de la satisfacción al cliente. }\end{array}$ \\
\hline
\end{tabular}




\begin{tabular}{|l|l||}
\hline Rodríguez, I., \& Alpuin, D. (2014) & $\begin{array}{l}\text { Desafía los modelos operativos tradicionales al introducir } \\
\text { un nuevo modelo, que concibe los procesos de negocio } \\
\text { como un activo estratégico clave para la organización. } \\
\text { Permite mejorar la asignación de recursos y realizar una } \\
\text { gestión consistente a lo largo de las diferentes funciones } \\
\text { desempeñadas en la organización. }\end{array}$ \\
\begin{tabular}{|l} 
Sánchez-Ruiz, Lidia \& Blanco, \\
Beatriz. (2015)
\end{tabular} & $\begin{array}{l}\text { Manera de organizar y gestionar un negocio dirigida a } \\
\text { todo tipo de empresas con la que se busca, simultánea- } \\
\text { mente, incrementar la eficiencia empresarial y aumentar } \\
\text { la satisfacción del cliente. }\end{array}$ \\
\hline $\begin{array}{l}\text { Medina, Nogueira \& Hernández } \\
\text { (2015) }\end{array}$ & $\begin{array}{l}\text { Herramienta para la concreción de las estrategias y el de- } \\
\text { sarrollo de la Mejora Continua con un enfoque holístico y } \\
\text { sistémico de la organización. }\end{array}$ \\
$\begin{array}{l}\text { Hernández Palma, H., Martínez } \\
\text { Sierra, D., \& Cardona Arbelaez, D. } \\
\text { (2016) }\end{array}$ & $\begin{array}{l}\text { Concepto moderno de direccionamiento, que ha resultado } \\
\text { como apoyo a la necesidad que tienen las organizaciones } \\
\text { de encaminar sus esfuerzos en la construcción de una } \\
\text { nueva estructura administrativa. }\end{array}$ \\
\hline $\begin{array}{l}\text { Zamora, M. H. (2017) } \\
\text { mán, L., \& Peralta Carrera, C. } \\
\text { (2018). }\end{array}$ & $\begin{array}{l}\text { Implica un avance lógico en las organizaciones hacia una } \\
\text { forma de enfocar la participación del personal y orientar } \\
\text { la misma hacia el cliente. } \\
\text { Permite una adecuada coordinación de todas las activida- } \\
\text { des y los recursos de la organización con vistas a lograr } \\
\text { los objetivos propuestos. }\end{array}$ \\
\hline $\begin{array}{l}\text { Mejora la interrelación y comunicación dentro de ella, } \\
\text { doniendo claro: qué se debe hacer, quién lo debe hacer, } \\
\text { Se evita la duplicidad de esfuerzos y que las acciones se } \\
\text { realicen de manera aislada }\end{array}$ \\
\hline
\end{tabular}

Fuente: Elaboración propia

Dentro de este orden de ideas, la gestión por procesos tiene como objetivo la creación de valor añadido para el cliente mediante el adecuado manejo de dichos procesos, en ese sentido, se la puede definir como un sistema de gestión, que interrelaciona sus actividades primarias y secundarias (Maldonado, 2013), considerando al proceso como elemento de encadenamiento a nivel estratégico, operativo y de 
apoyo (Zaratiegui, 1999) ello implica, mejorar o rediseñar dicho proceso, con el fin de transformar recursos (tangibles e intangibles) y entregar bienes o servicios de calidad, enfocado a satisfacer a los clientes.

Conforme en los argumentos teóricos recabados, se puede comprender que la gestión en las pequeñas y medianas empresas es afectada por cambios en el contexto político, económico, social, tecnológico, legal y ambiental, esto repercute en su arquitectura estructural formada por la estrategia, los procesos, como tambien insumos tecnológicos e informáticos; dentro de este marco la gestión por procesos contribuye a identificar objetivos de diseño y desarrollo de acciones que integren medidas de control, administrativas y de supervisión con el fin de orientar las actividades hacia los objetivos y metas institucionales, siempre considerando aquellas expectativas de cada cliente (interno/externo). Ante lo expuesto, una organización debe aprovechar la gestión por procesos como herramienta estratégica para diagramar los espacios o áreas (Ver figura 1) de la empresa, es claro, que el tamaño si influye en la cantidad de materias ha utilizar (Sotelo, 2016).

Figura 1.

Procesos de la organización

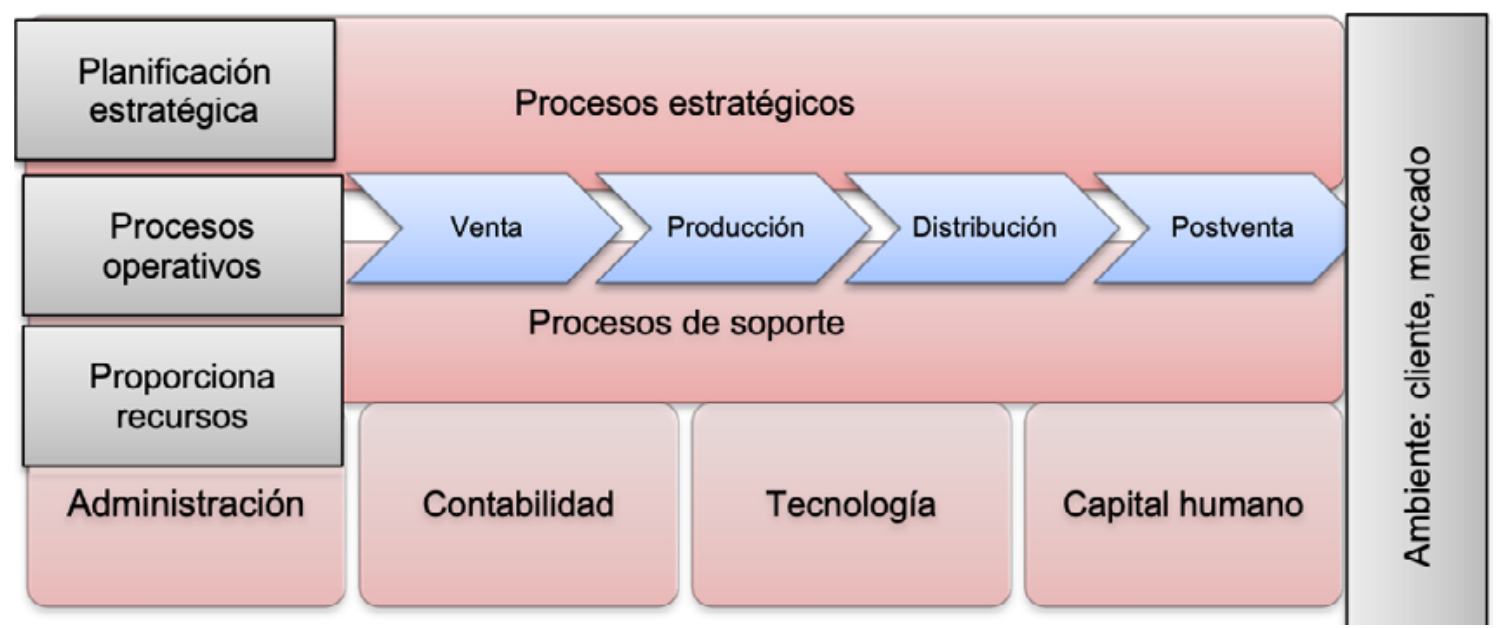

Fuente: Adaptado desde Barrios Hernandez y et al. (2019)

Por tal motivo, debe comprenderse que la efectividad de toda organización depende "de sus procesos organizacionales" (Medina et al., 2009, p. 66) así requerirá como principio, identificar aquellos procesos claves, que necesiten ser cambiados 
o rediseñados, pero que al final, el resultado indicará el desempeño alcanzado por las empresas agroindustriales como sistema holístico desde su estudio de la cadena de valor.

En relación con lo argumentado en párrafos anteriores, la gestión por procesos, como nuevo enfoque organizacional, constituye una herramienta estratégica que fundamenta sus postulados, en las formas de administrar, que incluye la identificación de los factores claves que pueden influir en el proceso de implementación y seguimiento, como: "la estrategia, la cultura, la estructura organizacional, la creación de valor, los procesos críticos” (Zamora, 2017, p. 25).

En ese sentido, SESCAM (2002) y Trkman (2009), hace énfasis en esta herramienta estratégica desde el estudio de la cultura organizacional como factor de éxito de la gestión por procesos.

La estrategia, se categoriza desde el pensamiento neoclásico, ahora el abordaje como factor empresarial constituye la manera en que una organización define los objetivos de la empresa, las acciones y recursos para cumplir con los objetivos (Sánchez \& Otros, 2019), en otras palabras, la administración toma decisiones que se materializan en estrategias de sostenibilidad de la gestión por procesos, por ende, es necesario dicha alineación (Pires \& Machado, 2006).

La cultura, es un factor que fortalece la estrategia empresarial desde liderazgo en costos y diferenciación (Villarreal \& Villareal, 2014), además subyace al ambiente interno de la realidad de cada empresa con iniciativa de adaptarse al cambio, lo que implica un patrón de comportamiento de los actores de la cadena, así tenemos: dirección de la organización; la reingeniería de los procesos; el tercer, corresponde a las acciones de los empleados, esta última es reflejo de su programación mental (Hofstede, 1980). Su aporte al estudio doctoral corresponde a comprender que la cultura en el contexto de las empresas pequeñas y medianas se manifiesta con patrones paternalistas (Casimiro \& Chambel, 2014).

La estructura organizacional, representa la estructura de los procesos y actividades de una organización (Matadamas et al., 2015) donde el recurso humano es clave por su nivel de adaptación, a pesar del esquema vertical establecido por la empresa.

Para el estudio, se debe comprender que la estructura organizacional no es definida por un organigrama, sino la forma como da respuesta a los cambios, en ello considera, responsabilidades, cliente, procesos claves, flujos de información y comunicación interna (Estupiñán \& Batista, 2018), entre otros indicadores. 
Los procesos críticos, son aquellos aspectos fundamentales de la organización, que permanentemente hay que hacerles un seguimiento para asegurar el rendimiento competitivo de la empresa (Rodríguez et al., 2004; Alfaro, 2009). En sentido, requiere de identificar dichos procesos, así, por ejemplo, los factores críticos del proceso productivo tienen que estar alineados con la gestión de los inventarios y la gerencia de los recursos humanos, por lo tanto,

La creación de valor es el efecto innovador que incursiona internamente en la empresa, con la finalidad de satisfacer la necesidad del cliente, su materialización corresponde a la ventaja competitiva (Porter, 1987) a través del encadenamiento de las actividades de los procesos de la cadena de valor. en ese sentido, se crea la necesidad de diseñar instrumentos que a más de estandarizar los procesos (Huapaya, 2019) permita evaluar y dar seguimiento, siendo importante la creación e indicadores de eficacia y eficiencia (Alfaro, 2009).

La aplicación de la gestión por procesos como herramienta estratégica en una empresa contribuye la comprensión de "los procesos de negocios, sus fortalezas y debilidades” (Medina et al., 2009) así también, la reducción de errores, eliminación de las "ineficiencias asociadas a la repetitividad de acciones o actividades, las mismas que ocasionan desperdicio de recursos por asignación inapropiada.

En criterio adverso, Tatić, Haračić \& Haračić (2018) sostiene que la gestión inadecuada de los procesos y el insuficiente enfoque sistemático crean procesos innecesarios, aumentan la ineficiencia y reducen la competitividad, lo que en última instancia afecta la capacidad de la empresa para operar con éxito, dentro de un corto o largo plazo. En ese sentido, no solo se busca la eficiencia de los procesos, sino la eficacia de su gestión con la finalidad de satisfacer las necesidades y expectativas de los clientes, esta puede incluir su fase de automatización donde se modela y simula procesos, se valida flujos y procesos, además se asigna tiempo a cada actividad o tarea en el proceso.

En ese sentido, el autor Sánchez-Ruiz $(2015)^{3}$ presenta un estudio sobre las razones que motivan a las empresas a implantar la gestión por procesos. Este autor aclara, que su adopción mejoraría los flujos de información y la generación de información necesaria para comprender el producto de entrada y salida, generado

\footnotetext{
${ }^{3 *}$ Sánchez-Ruiz, L. (2015). Análisis de las razones para implantar la gestión por procesos mediante la teoría de medición de Rasch. (September). p.485-486
}

Gestión por procesos como herramienta estratégica en las pequeñas y medianas empresas 
en cada uno de los procesos organizacionales, lo que significa, aumentos de productividad o mejora del valor ofrecido al cliente, como parte de la mejora interna. A lo expuesto, el principal impacto de las pequeñas y medianas empresas esta en su relevancia en el crecimiento y desarrollo de la economía del país, debido a la generacion de empleo, y con ello el eincremento de la competitividad que interrelaciones actuaciones de agentes económicos como el Estado, universidad, organizaciones No gubernamentales.

Así también, existen estudios (Davenport \& Short, 1990; Armistead \& Machin, 1998; Armistead \& Pritchard, 1998; Hung, 2006; Palmberg, 2010) referentes a las motivaciones para implantar la gestión por procesos, como es el añadir valor al cliente, aumento de la competencia nacional y/o internacional, mejora de la efectividad organizacional, barreras en la diversificación de tareas y funciones, aumento de la calidad del aprendizaje colectivo dentro de la organización y entre la organización y su entorno, la adaptación de estructuras funcionales a los cambios del ambiente, el aumento de productividad, y la falta de calidad, estandarización de los procesos.

\section{Resultados y discusión}

Como se expuso en el apartado de métodos y materiales, el presente producto aborda aspectos teóricos sobre la gestión por procesos, parten de la reflexión final que formará parte del corpus referencial del proyecto de investigación; por otra parte, se da respuesta a las interrogantes de revisión ¿Qué se ha tratado sobre la gestión por procesos? ¿Qué aspectos de la gestión por procesos le otorgan la importancia como herramienta estratégica?

Los resultados del recorrido teórico sobre la gestión por procesos aproximan a su importancia en un entorno dinámico, que fracciona pensamientos tradicionales asociados a dimensiones tecnológicas y de productividad. Para inicios del siglo XXI este nuevo enfoque toma importancia no solo al procedimentar los procesos, sino gestionarlos con buenas prácticas vinculadas a la mejora continua y satisfacción de cada cliente (interno/externo). En el caso de las pequeñas y medianas empresas se ha propuesto mejorar sus procesos desde una visión integradora que los alinee con la estrategia, misión y objetivos, esto implica una innovación interna en la gestión 
de las estructuras funcionales que son limitadas por problemas a nivel estratégico, operativo y de apoyo como la informalidad, capacidad gerencial, así también dificultad de acceso a financiamiento. Así mismo, se destaca los aportes relevantes explícitamente como en la coordinación de tareas y actividades, que técnicamente se materializa en los procesos agregadores de valor.

La gestión por procesos es reconocida a nivel internacional por su orientación a la satisfacción del cliente y agrupa principios tales como: responsabilidad social, planificación estratégica, alineamiento de intereses, orientación al cliente y análisis de riesgos para el alcance de la excelencia empresarial (Cabrera et al., 2015). En ese sentido, aparece como sistema que mejora la eficiencia interna y elimina lo que no da valor al cliente interno/externo (Sánchez \& Blanco, 2014).

Con base a lo concluido por Medina et al., (2009) queda por comprobar en la investigación de campo de la tesis doctoral, el nivel de conciencia por parte de los empresarios al aplicar las buenas prácticas de gestión, esto refiere a comprender que el logro de la eficiencia y eficacia dependen del estado de los procesos de las pequeñas o medianas empresas. Por otra parte, relacionar aquellos elementos motivadores expuestos por Barrios Hernandez et al., (2018) vienen impulsando la implementación de la gestión por procesos, así tenemos: la necesidad de mejorar en eficiencia y productividad; el logro de objetivos empresariales; la mejora de imagen corporativa, potencialización de mercado y el fortalecimiento de la competitividad.

Finalmente, las bondades expuestas anteriormente, son amenazadas por factores que le afectan como: recurso humano, cultura, innovación. Así también la mejora continua, la innovación, aprendizaje, el conocimiento, experiencia, reputación, recursos y capacidades, estrategias, y el prestigio de la empresa, son factores internos que apoyan a enfrentar los efectos de los factores externos. En ese sentido, queda por comprobar si la gestión por procesos es una herramienta estratégca efectiva para el logro de la mejora continua y la materialización de los objetivos organizacionales. 


\section{Conclusiones}

Entre las conclusiones se establece que la gestión por procesos como herramienta estratégica gana importancia en las pequeñas y medianas empresas por la organización lógica de sus recursos, su incorporación es adaptable en estructuras tradicionales porque establece una gestión interfuncional generadora de valor para cada cliente (interno/externo), esto ha conllevado a materializar los objetivos institucionales, determinación de entradas y salidas de los procesos estratégicos, operativos y de apoyo, esto significa aprovechar la disminución de actividades que no aporten a la eficiencia interna y eliminar todo aquello que el cliente no valora.

En el mismo marco, la gestión por procesos corresponde a un sistema integral; su visión holística y sistémica (Medina et al., 2009; Sánchez \& Blanco, 2014) permite a las partes responsables del proceso de negocio comprender que no toda actividad realizada en las pequeñas y medianas empresas es estándar, asi por ejemplo, en un ente del sector secundario de la economía el transformar materia prima no debe ser tratada como único proceso (SESCAM, 2002), porque existen otras actividades, tareas, procedimientos que requieren de la aplicación estratégica de la gestión por procesos, pues esto conlleva un conjunto de elementos de entrada y salida (recursos físicos, métodos, costos, personas); dichos elementos interrelacionados facilitan a la empresa su posicionamiento en el mercado, y comprender mejor los complejos vínculos entre la satisfacción del cliente a través del "desempeño general de los procesos y la productividad” (Armistead \& Machin, 1998, p. 335).

En la aplicación de la técnica de lectura, se apreció limitados aportes de investigadores sobre la gestión por procesos en pequeñas y medianas empresas, la evolución del pensamiento administrativo, se ha enmarcado de forma representativa desde las grandes empresas; sin embargo, los resultados, permiten generalizar hasta este pequeño grupo de la economía (PyMEs) que enfrenta la resistencia al cambio, estrategias ambiguas, estancamiento de tecnologías, directivos rígidos, falta de compromiso a largo plazo y desconocimiento de actividades de planificación; dichos hallazgos encontrados, requieren ser desafiados con herramientas estratégicas que no se enfoquen en solo en tareas, oficios, personas, estructura, sino también en los procesos. Por ende, se reafirma la necesidad de adoptar la gestión por procesos en estas organizaciones desde nuevas concepciones de encadenamiento que permita identificar y desechar aquellos procesos que no agreguen valor a las empresas. 
Para futuras investigaciones, se puede considerar los vacios del recorrido teórico sobre la aplicabilidad de la gestión por procesos en pequeñas y medianas empresas con un enfoque hacia su encadenamiento de valor, esto hace útil la adaptación de un mapa de procesos que permita aquellas economías tradicionales de estructura familiar (PyMEs) aumentar su capacidad de respuesta en el mercado.

\section{Referencias bibliográficas}

Armistead, C., \&Machin, S. (1998). Business process management: implications for productivity in multi-stage service networks. International Journal of Service Industry Management, 9(4), 323-336. doi:10.1108/09564239810228849

Avolio, B., Mesones, A., \& Roca, E. (2011). Factores que limitan el crecimiento de las micro y pequeñas empresas en el Perú (MYPES). Strategia, (22), 70-80.

Barrios-Hernández, K., Contreras-Salinas, J. \& Olivero-Vega, E. (2019). La Gestión por Procesos en las Pymes de Barranquilla: Factor Diferenciador de la Competitividad Organizacional. Información tecnológica, 30(2), 103-114. https:// dx.doi.org/10.4067/S0718-07642019000200103

Beltrán, A. (2006). Los 20 problemas de la pequeña y mediana empresa. Sotavento MBA, (7), 8-15.

Bravo, J. (2011). Gestión de Procesos (Valorando la práctica). Evolución Editorial.

Cabrera, Henrry R,; Medina León, A,; Nogueira Medina, D. y Núñez Chaviano, Q. (2015). Revisión del estado del arte para la gestión y mejora de los procesos empresariales. Enfoque UTE, 6(4),1-22. [fecha de Consulta 3 de Julio de 2020]. ISSN: Disponible en: https://www.redalyc.org/articulo.oa?id=5722/572260849001

Davenport, T., \& Short, J. (1990). The New Industrial Engineering: Information Technology And Business Process R. Management, 31(4), 11-27. Retrieved from http://scholar.google.com/scholar?hl=en\&btnG=Search\&q=intitle:The+New+Industrial+Engineering:+Information+Technology+and+Business+Process+Redesign $\#$ 7 (p.20) 
Estupiñán Ricardo, Jesús \& Batista Hernandez, Noel. (2018). Gestión Empresarial y Posmodernidad. Disponible en: https://www.researchgate.net/publication/339630971_Gestion_Empresarial_y_Posmodernidad.

García, J., Cazallo, A., Barragan, C. E., Mercado, M., Olarte, L., \& Meza, V. (2019). Indicadores de Eficacia y Eficiencia en la gestión de procura de materiales en empresas del sector construcción del Departamento del Atlántico, Colombia. Revista ESPACIOS, 40(22). Disponible en: http://www.revistaespacios.com/ a19v40n22/19402216.html

González, A., Leal, L., Martínez, D., Morales, D. (2019). Herramientas para la gestión por procesos. Cuadernos Latinoamericanos de Administración, XV(28). https://www.redalyc.org/articulo.oa?id=409659500003

González, A., Villamil, D. \& Arteaga, W.(2019). Propuesta teórica de mapa de procesos sustentables para crear valor en PYMES. Revista de divulgación y tecnología de la Red Internacional de Investigadores de Ingeniería Industrial<REDI4> 5(10).

Harrington, H. J. (1991). Improving business processes. The TQM Magazine, 3(1). doi: $10.1108 /$ eb059514

Hernández Palma Hugo, Barrios Parejo Ignacio, Martínez Sierra David (2018). Gestión de la calidad: elemento clave para el desarrollo de las organizaciones. Criterio Libre, 16 (28), 179-195 ISSN 1900-0642

Hung, R. (2006). Business process management as competitive advantage: A review and empirical study, Total Quality Management \& Business Excellence, $17(1), 21-40$.

Lange, Ivonne (2013). Consideration of sustainable development principles in process management. PM World Journal, Vol. II, Issue VI - June 2013. PROJECT MANAGEMENT

Maldonado Mina, D. O. (2013). Diseño de un modelo de gestión por procesos para mejorar la producción de borregos africanos en la Asociación de Producción Agropecuaria "Los Salinenses". La Universidad Técnica Particular de Loja

Mallar, M. (2010). La Gestión por Procesos: un enfoque de gestión eficiente. Visión de futuro, 13(1) http://www.scielo.org.ar/scielo.php?script=sci_arttext\&pi$\mathrm{d}=\mathrm{S} 1668-87082010000100004 \& \operatorname{lng}=$ es\&tlng=es. 
Medina León, A.; Nogueira Rivera. D.; Hernández-Nariño, A. y Comas Rodríguez, Raúl. (2019). Procedimiento para la gestión por procesos: Métodos y herramientas de apoyo. Ingeniare. Revista chilena de ingeniería, vol. $27 \mathrm{~N}^{\circ} 2$, pp. 328-342 https://scielo.conicyt.cl/pdf/ingeniare/v27n2/0718-3305-ingeniare-27-02-00328.pdf

Medina León, A.; Nogueira Rivera, D. y Hernández Nariño, A. (2009). Relevancia de la gestión por procesos en la planificación estratégica y la mejora continua. Eídos, (2), 65-72.

Medina, A., Nogueira, D., \& Hernández, A. (2002). Relevancia de la Gestión por Procesos en la Planificación Estratégica y la Mejora Continua. EíDOS.

Medina León, A. Nogueira Rivera, D. Hernández Nariño, A. (2015). Relevancia de la gestión por procesos en la planifi- cación estratégica y la mejora continua. Revista Eídos. Recuperado de: http://www.altagestion.com.co/boletines/mai- ling2015/ redes_sociales/doc/0702_DOCUMENTO_RelevanciaGPP_20150708.pdf

Medina, A.; Nogueira, D.; Hernández, A., \& Díaz, Y. (septiembre-diciembre de 2012). Consideraciones y criterios para la selección de procesos para la mejora: Procesos Diana. Ingeniería Industrial, XXXIII (3), 272-2811.

Mila-Carvajal, F., Reyes-Ordoñez, B., Dueñas-Mendoza, A. \& Armas-Arias, M. (2019). Gestión por Procesos en las Micro, Pequeñas y Medianas Empresas comerciales de la ciudad Esmeraldas, Ecuador. Veritas \& Research, 1(2), 140 -149 .

Ochoa Baque, A., Herrera Leon, J., \& Mosqera Aguirre, J. (2012). Diseño de un sistema de gestión por procesos para una empresa dedicada a brindar servicios medicos ubicada en la ciudad de Guayaquil. (1), 1-8. Retrievedfrom https:// www.dspace.espol.edu.ec/.../Datos adjuntos sin titulo\%25...

Palmberg, K. (2010) "Experiences of implementing process management: A multiple- case study”, Business Process Management Journal, 16(1), 93, 2010.

Porter, M. (1987 ed. rev.2006) Ventaja Competitiva. Creación y sostenimiento de un desempeño superior. CECSA Editorial. México. 547

Ricardo, H., Medina, A., Nogueira, D. \& Núñez, Q. (2015). Revisión del estado del arte para la gestión y mejora de los procesos empresariales. Enfoque UTE, 6(4), 1-22. https://doi.org/10.29019/enfoqueute.v6n4.75

Gestión por procesos como herramienta estratégica en las pequeñas y medianas empresas 
Rodríguez, I., \& Alpuin, D. (2014). La Gestión por Procesos en las Organizaciones. La forma en la que los resultados se logran (p. 10). p. 10. Retrievedfromhttps:// www2.deloitte.com/content/dam/Deloitte/uy/Documents/strategy/Gestión por procesos para web.pdf

Sánchez, L. \& Blanco, B. (2014). La Gestión por Procesos: un campo por explorar. Dirección y Organización, 54, 54-71.

SESCAM. (2002). La Gestión por Procesos. Recuperado a partir de www.chospab. es/calidad/UCalidad/Documentos/Gestiondeprocesos

Sotelo Raffo, J. L. F. (2016). La gestión por procesos en su papel de estrategia generadora de ventaja competitiva aplicada a los enfoques de asociatividad de las MyPES: caso peruano (Tesis doctoral, Universitat Politècnica de Catalunya). Repositorio Institucional. https://www.tesisenred.net/bitstream/handle/10803/406961/TJLFSR1de1.pdf?Seque

Tatić, K., Haračić, M. \& Haračić, M. (2018). The improvement of business efficiency through Business process management, Economic Review - Journal of Economics and Business (Vol. XVI, Issue 1.). Tuzla, BiH. 31-43

Zamora, M. H. (2017). Teoría de la gestión por procesos: un análisis del Centro de Fórmulas Lácteas Infantiles del Hospital Sor María Ludovica de La Plata. (Tesis de maestría, Universidad Nacional de La Plata). Repositorio Institucional. Retrievedfromhttp://sedici.unlp.edu.ar/handle/10915/61203

Zaratiegui, J. (1999). La gestión por procesos: su papel e importancia en la empresa. Economía Industrial, (330), 81-88. 
Universidad Técnica de Machala

Dirección de Investigación

\section{Editorial UTMACH}

https://investigacion.utmachala.edu.ec/portal/

Primera edición 2021

Colección Semana de la Ciencia

PDF interactivo 



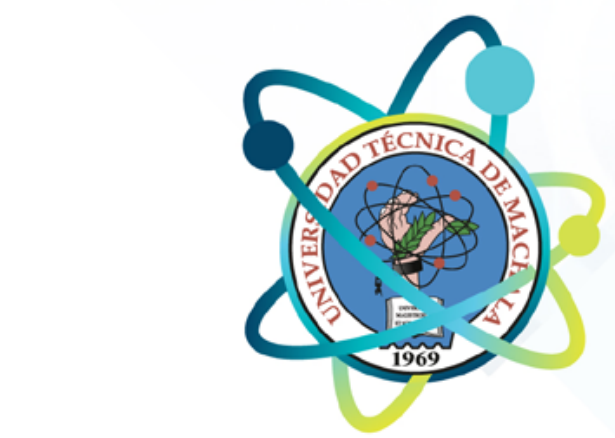

\section{Investigación UTMACH}

2021

Km. 5 1/2 Machala - Pasaje

Teléfono: (593) 72933345 Ext. 173

E-mail: editorial@utmachala.edu.ec investigacion.utmachala.edu.ec 